Supporting Information for

\title{
Synthesis of Monodispersedly Sized ZnO Nanowires from Randomly Sized Seeds
}

Xixi Zhao ${ }^{1}$, Kazuki Nagashima ${ }^{1,2^{*}}$, Guozhu Zhang ${ }^{1}$, Takuro Hosomi ${ }^{1,2}$, Hideto Yoshida ${ }^{3}$, Yuya Akihiro ${ }^{1}$, Masaki Kanai ${ }^{1}$, Wataru Mizukami ${ }^{4}$, Zetao Zhu ${ }^{5}$, Tsunaki Takahashi ${ }^{1,2}$, Masaru Suzuki ${ }^{1}$, Benjarong Samransuksamer ${ }^{1}$, Gang Meng ${ }^{6}$, Takao Yasui ${ }^{2,5}$, Yuriko Aoki $^{4}$, Yoshinobu Baba ${ }^{5}$ and Takeshi Yanagida ${ }^{1^{*}}$

${ }^{1}$ Institute for Materials Chemistry and Engineering, Kyushu University, 6-1 Kasuga-Koen, Kasuga, Fukuoka 816-8580, Japan

${ }^{2}$ Japan Science and Technology Agency (JST), PRESTO, 4-1-8 Honcho, Kawaguchi, Saitama 332-0012, Japan

${ }^{3}$ The Institute of Scientific and Industrial Research, Osaka University, 8-1 Mihogaoka, Ibaraki, Osaka 567-0047, Japan

${ }^{4}$ Interdisciplinary Graduate School of Engineering Sciences, Kyushu University, 6-1 Kasuga-Koen, Kasuga, Fukuoka 816-8580, Japan

${ }^{5}$ Department of Biomolecular Engineering, Graduate School of Engineering, Nagoya University, Furo-cho, Chikusa-ku, Nagoya 464-8603, Japan

${ }^{6}$ Anhui Provincial Key Laboratory of Photonic Devices and Materials, Anhui Institut of Optics and Fine Mechanics, Chinese Academy of Sciences, Hefei 230031, China 
Corresponding author's e-mail: kazu-n@cm.kyushu-u.ac.jp, yanagida@cm.kyushuu.ac.jp 

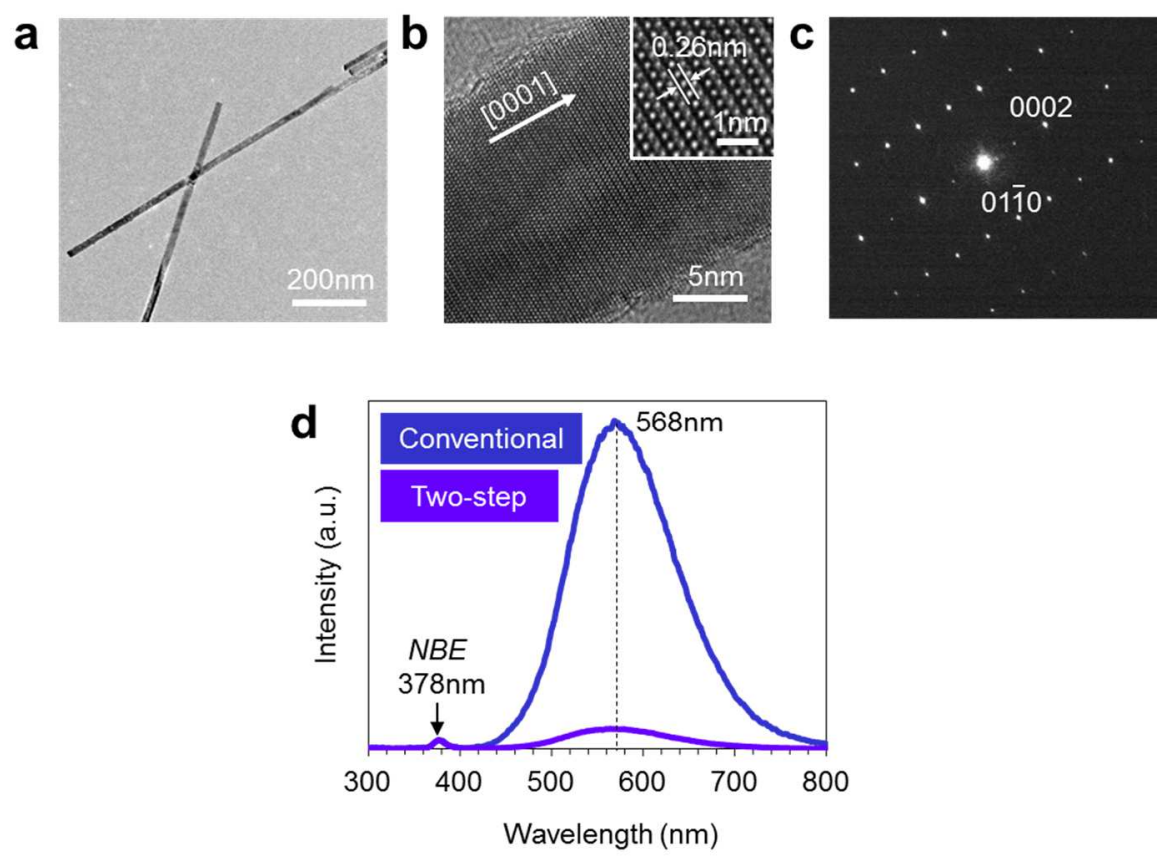

Figure S1. The characterization of the two-step grown $\mathrm{ZnO}$ nanowires. (a) Low magnification TEM image, (b) high magnification TEM image, and (c) selected area electron diffraction (SAED) pattern of the two-step grown $\mathrm{ZnO}$ nanowires. Inset of (b) shows the atomically resolved TEM image. For the two-step grown nanowires, step 1 with $\mathrm{NH}_{3} 50 \mathrm{mM}, C_{Z n} 2.5 \mathrm{mM}$ for $3 \mathrm{~h}$, and step 2 with no $\mathrm{NH}_{3}, C_{Z n} 5 \mathrm{mM}$ for $12 \mathrm{~h}$ are utilized. The TEM analysis represents that the fabricated nanowires are single crystalline with a growth direction along [0001], which is consistent with the conventional hydrothermally grown $\mathrm{ZnO}$ nanowires. (d) Room temperature photoluminescence spectroscopy data of two-step grown $\mathrm{ZnO}$ nanowires. The wavelength of excitation light is $256 \mathrm{~nm}$. As a comparison, the data of single-step grown $\mathrm{ZnO}$ nanowires (no $\mathrm{NH}_{3}, C_{\mathrm{Zn}}$ $25 \mathrm{mM}$ for $12 \mathrm{~h}$ ) is shown. Two peaks at $378 \mathrm{~nm}$ and $568 \mathrm{~nm}$ are observed, which are respectively corresponding to near-band edge (NBE) emission and the emission related to crystal imperfections (e.g. oxygen vacancies, Zn interstitials, surface state). The data shows that the crystal imperfections in the two-step grown nanowires are less than that of 
conventional $\mathrm{ZnO}$ nanowires. The better crystal quality of the two-step grown nanowires than the single-step grown ones might result from the suppressed nucleation on $(10 \overline{1} 0)$ plane. Watanabe et al. ${ }^{[\text {Ref] }}$ demonstrated that more crystal imperfections are induced on (1010) plane rather than on (0001) plane in the hydrothermal growth of $\mathrm{ZnO}$ nanowires. Since the single-step growth is conducted at $C_{Z n} 25 \mathrm{mM}$, where it is above the critical concentration for nucleation on (10 $\overline{1} 0)$ plane, the plenty of crystal imperfections could be induced into the nanowires. On the contrary in the two-step grown nanowires, the nucleation on (10 $\overline{1} 0)$ plane is precisely suppressed by controlling $C_{Z n}$, leading to the better crystal quality as seen in PL spectrum.

[Ref] Watanabe, K.; Nagata, T.; Oh, S.; Wakayama, Y.; Sekiguchi, T.; Volk, J.; Nakamura, Y. Arbitrary cross-section SEM-cathodoluminescence imaging of growth sectors and local carrier concentrations within micro-sampled semiconductor nanorods. Nat. Commun. 2016, 7, 10609. DOI: 10.1038/ncomms10609 

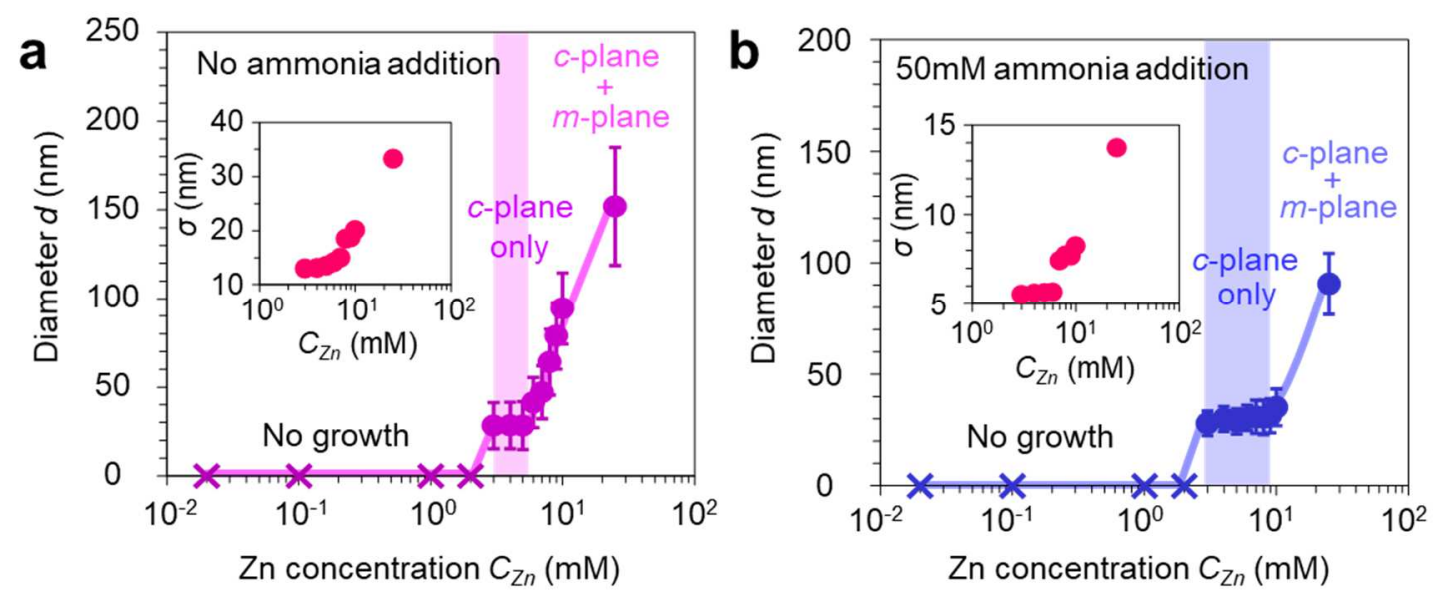

Figure S2. Zn concentration dependence on the diameter of single-step grown $\mathrm{ZnO}$ nanowires with (a) no ammonia addition and (b) $50 \mathrm{mM}$ ammonia addition. Insets show the standard deviation value of nanowire diameter. The growth time is constant to be 12 h. When increasing the $\mathrm{Zn}$ concentration, the nanowire growth is fist observed at above $3 \mathrm{mM}$ in (a) and (b) with maintaining their diameter. Further increase of the $\mathrm{Zn}$ concentration above $5 \mathrm{mM}$ in (a) and $9 \mathrm{mM}$ in (b) leads to the increase of diameter. These two threshold concentrations correspond to the critical nucleation concentrations on (0001) plane (c-plane) and (10 $\overline{1} 0)$ plane (m-plane). The concentration range between these two threshold values promotes the anisotropic growth of $\mathrm{ZnO}$ nanowires by suppressing the sidewall growth. These threshold values are influenced by various parameters such as capping agent, surface potential at given $\mathrm{pH}$ condition and supersaturation degree in growth solution. As seen in the insets, the minimum diameter distributions of the nanowires are given at the condition where the sidewall growth is suppressed. However, the narrow diameter distribution of the two-step grown nanowires in figure 1 (a) cannot be achievable by solely controlling the precursor concentration in single-step growth. 
Crystal plane dependent critical concentrations for a nucleation: In the nucleation theory, the nucleation phenomenon is dominated by adsorption and desorption of atoms. When increasing the precursor concentration, the adsorption rate of atoms increases. Since the nucleation in solution occurs when the size of nuclei exceeds the critical nucleation size by the sufficient precursor supply, the precursor concentration is one of the most important parameter for controlling nucleation event. For a nucleation in the presence of solid surface, an interaction between a nucleus and a solid surface should be taken into account with free energy barrier for a nucleation. The presence of solid surface significantly reduces the free energy barrier for a nucleation since an interaction between a nucleus and a solid surface is much stronger than that between a nucleus and liquid. If a surface energy (an interfacial energy in solution system) is different among crystal planes, the free energy barrier for a nucleation on each crystal plane should be different. In the $\mathrm{ZnO}$ crystal, (0001) polar plane has higher surface energy compared with (10 $\overline{1} 0)$ plane. In general, the free energy barrier for a nucleation becomes lower on a crystal plane with larger surface energy. Therefore, the critical concentration for nucleation on (0001) plane is lower than that on $(10 \overline{1} 0)$ plane. Although the interfacial energy in solution system differs from the surface energy, the interfacial energy on (0001) plane remains higher than that on $(10 \overline{1} 0)$ plane by considering the preferential crystal growth on $(0001)$ plane in the fabricated nanowires. Based on this principle, when increasing the $\mathrm{Zn}$ precursor concentration, none of nanowire growth is first observed under the critical concentration on (0001) plane and then the preferential crystal growth on (0001) plane is observed in the concentration window where is above the critical concentration on (0001) plane and below that on (1010) plane. By further increasing the $\mathrm{Zn}$ precursor concentration over the critical concentration on (1010) plane, the crystal growth both on 
(0001) plane and (1010) plane. Thus the $\mathrm{Zn}$ precursor concentration can control the competitive nucleation event on (0001) plane and (1010) plane, which is important to design the anisotropic crystal growth of $\mathrm{ZnO}$ nanowires. 

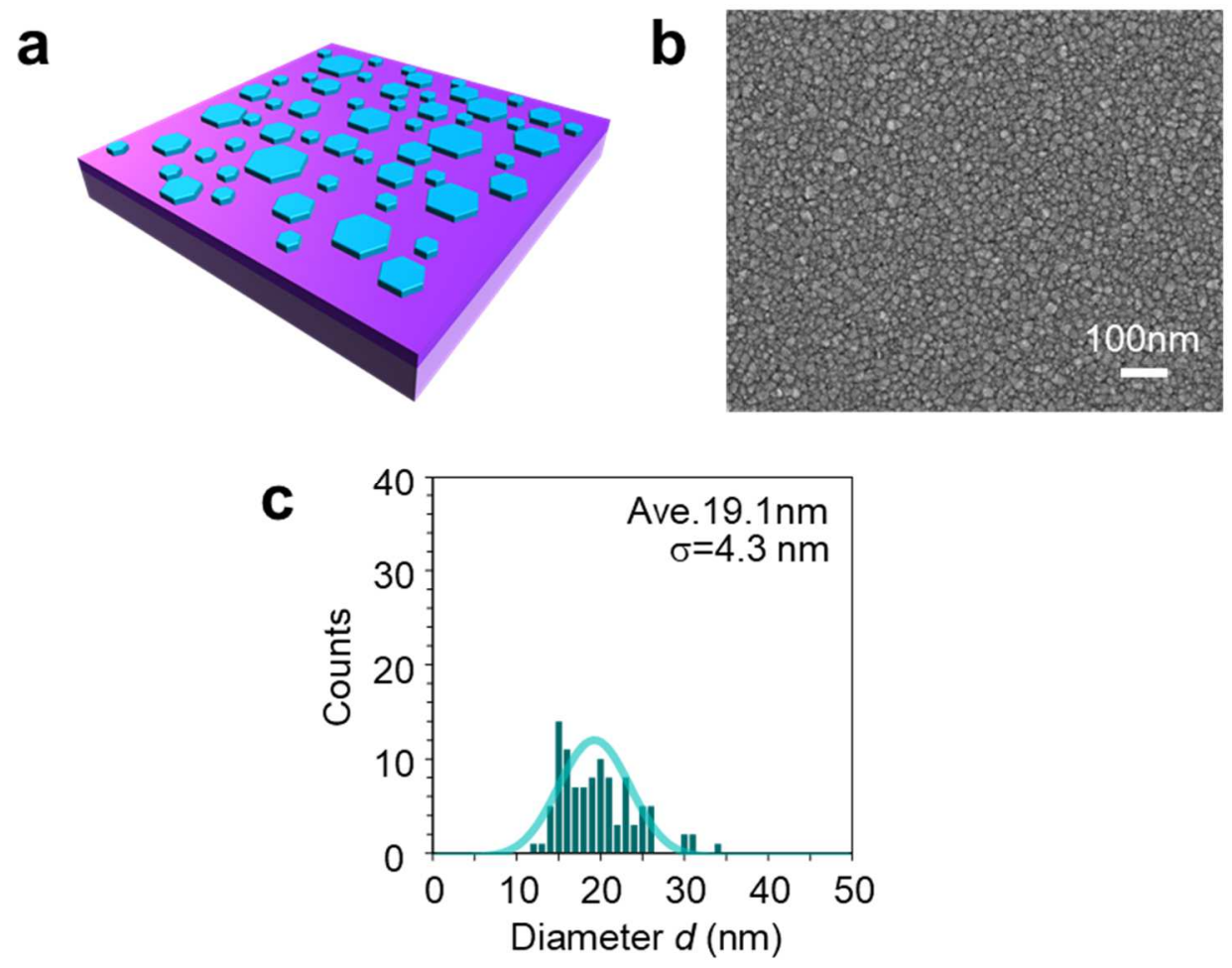

Figure S3. (a) Schematic image, (b) FESEM image and (c) size distribution data of the $\mathrm{ZnO}$ seed layer deposited onto $\mathrm{SiO}_{2} / \mathrm{Si}$ substrate by RF sputtering. The thickness of seed layer is about $30 \mathrm{~nm}$. In the FESEM image, the densely deposited grains are seen. The size distribution of $\mathrm{ZnO}$ seed layer is wider than that of two-step grown monodispersedly sized $\mathrm{ZnO}$ nanowires shown in figure 1 (a). 

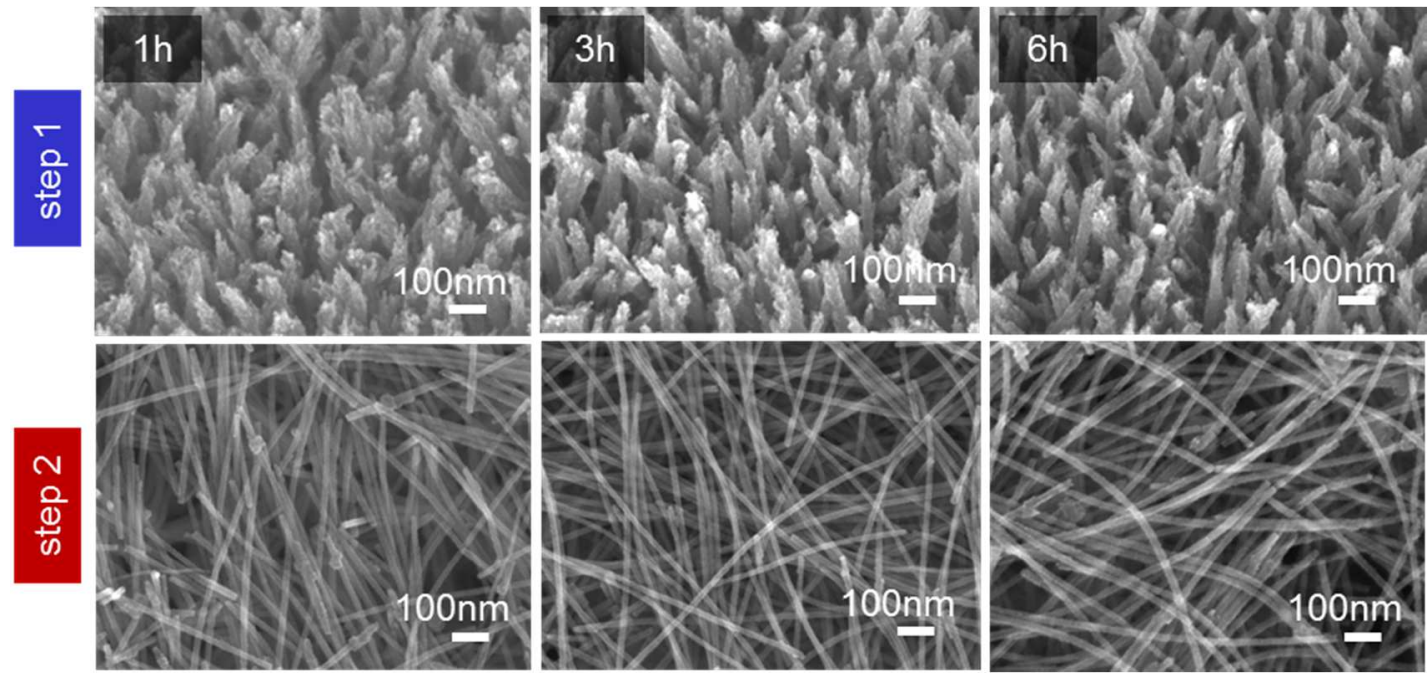

Figure S4. The growth time dependence on the morphologies of the two-step grown $\mathrm{ZnO}$ nanostructures/nanowires observed after step 1 (upper) and two-step growth with step 2 (lower). In this experiment, only the growth time of step 1 is varied (1 h, $3 \mathrm{~h}, 6 \mathrm{~h})$ and the other conditions are kept constant $\left(\mathrm{NH}_{3} 50 \mathrm{mM}, C_{Z n} 2.5 \mathrm{mM}\right.$ for step 1 and no $\mathrm{NH}_{3}, C_{Z n}$ $5 \mathrm{mM}$ for step 2). The growth time of step 2 is $12 \mathrm{~h}$. 


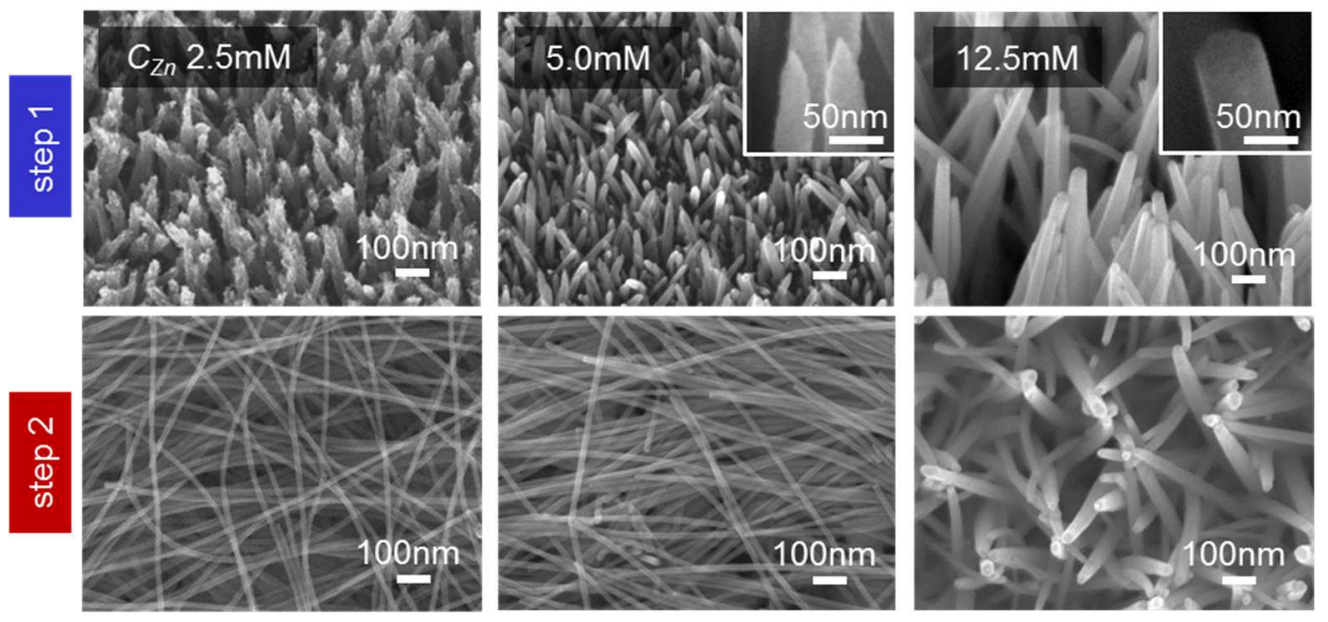

Figure S5. The $\mathrm{Zn}$ concentration dependence on the morphologies of the two-step grown $\mathrm{ZnO}$ nanostructures/nanowires observed after step 1 (upper) and two-step growth with step 2 (lower). In this experiment, only the $\mathrm{Zn}$ concentrations of step 1 is varied $(2.5 \mathrm{mM}$, $5.0 \mathrm{mM}, 12.5 \mathrm{mM})$ and the other conditions are kept constant $\left(\mathrm{NH}_{3} 50 \mathrm{mM}, 3 \mathrm{~h}\right.$ for step 1 and no $\mathrm{NH}_{3}, C_{Z n} 5 \mathrm{mM}, 12 \mathrm{~h}$ for step 2). Insets show the magnified images at the tip of nanostructures/nanowires. Clearly, the secondary nanowire growth is observed only when the sharp-tips are constructed by step 1 and no longer observable from the flat-tips. 


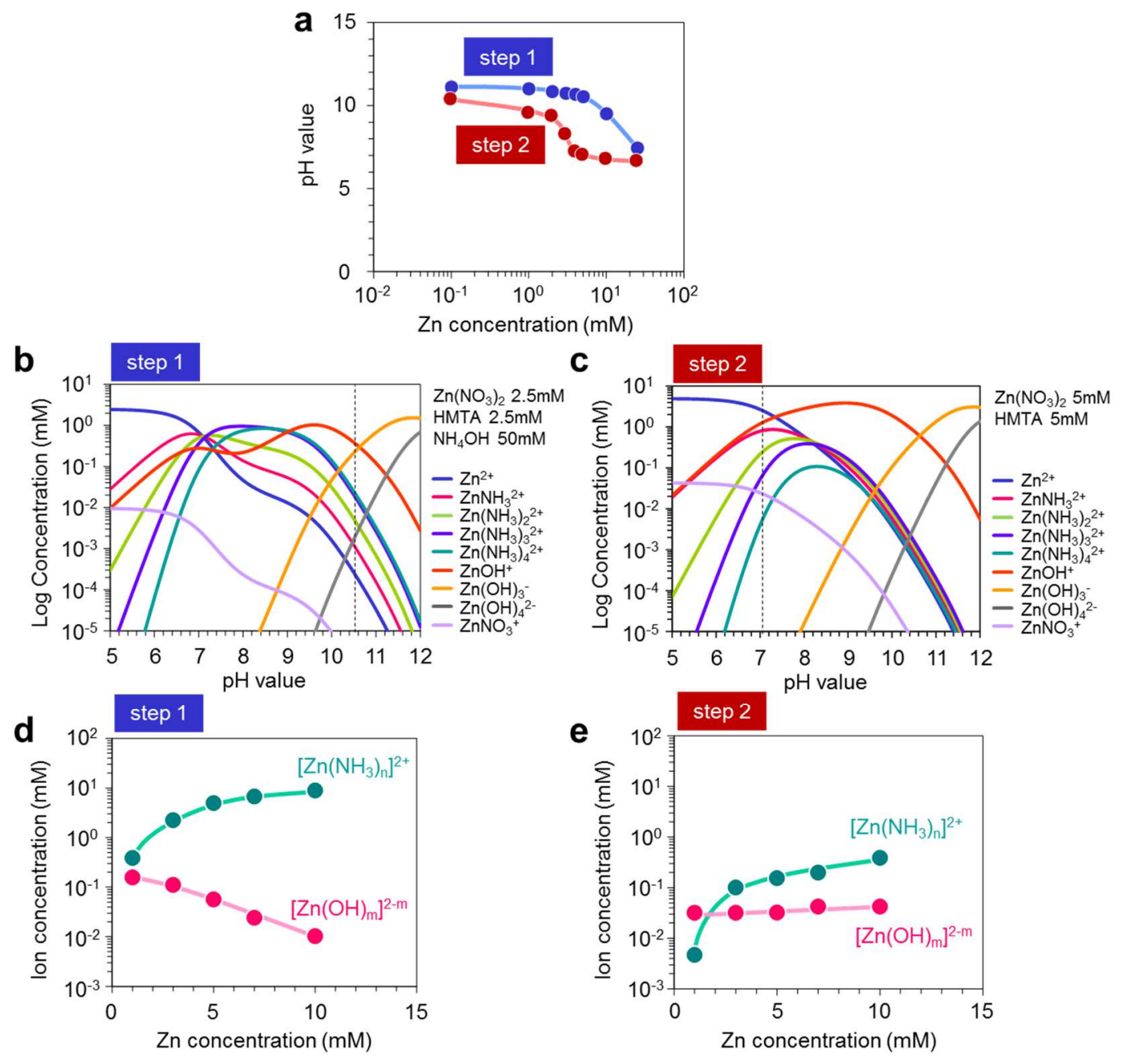

Figure S6. (a) The experimentally measured $\mathrm{pH}$ values of the solutions for step 1 and step 2 when varying the $\mathrm{Zn}$ concentration. $(\mathrm{b}, \mathrm{c})$ The calculated data of $\mathrm{pH}$ dependent equilibrium concentrations of ionic species existing in the growth solutions of (b) step 1 and (c) step 2. The dash lines show the experimentally measured $\mathrm{pH}$ values for each condition. (c,d) The $\mathrm{Zn}$ concentration dependence on the ion concentrations of $\left[\mathrm{Zn}\left(\mathrm{NH}_{3}\right)_{\mathrm{n}}\right]^{2+}$ and $\left[\mathrm{Zn}(\mathrm{OH})_{\mathrm{m}}\right]^{2-\mathrm{m}}$ species for the growth solutions of (d) step 1 and (e) step 2 , respectively. The ion concentrations were calculated by using the measured $\mathrm{pH}$ values. 

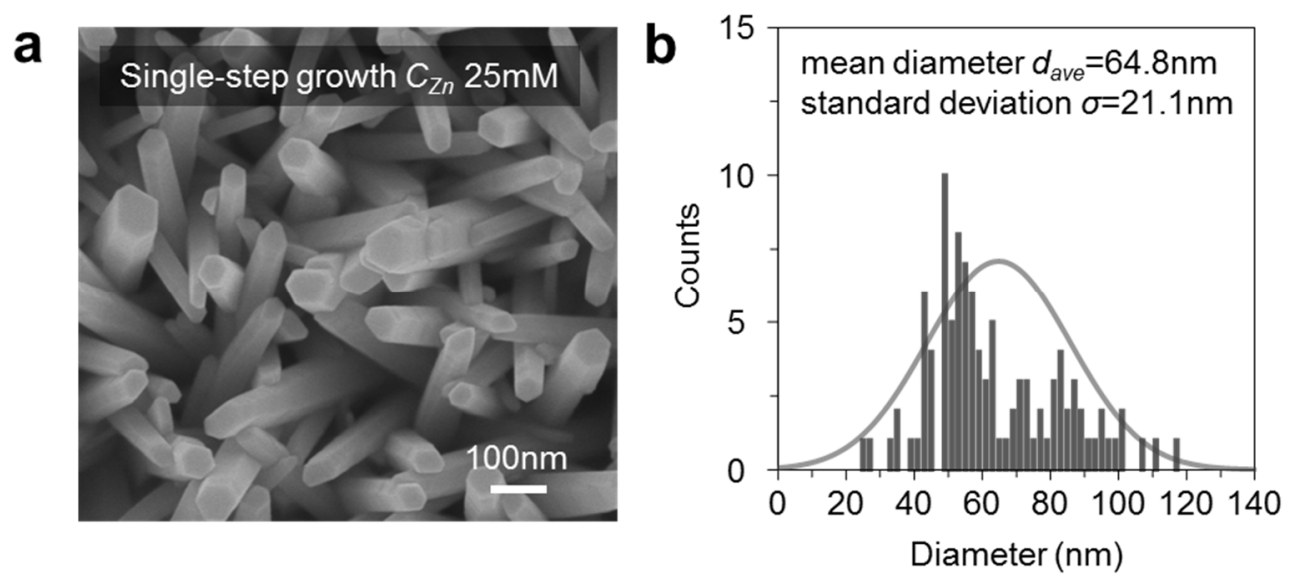

Figure S7. (a) Typical FESEM image and (b) diameter distribution data of the flat-top $\mathrm{ZnO}$ nanowires grown on $\mathrm{Si}$ substrate by the single-step growth with $C_{Z n} 25 \mathrm{mM}$. In this growth, no $\mathrm{NH}_{3}$ is added. The growth time is $12 \mathrm{~h}$. The mean diameter $d_{\text {ave }}$ and the standard deviation value $\sigma$ are also shown. 

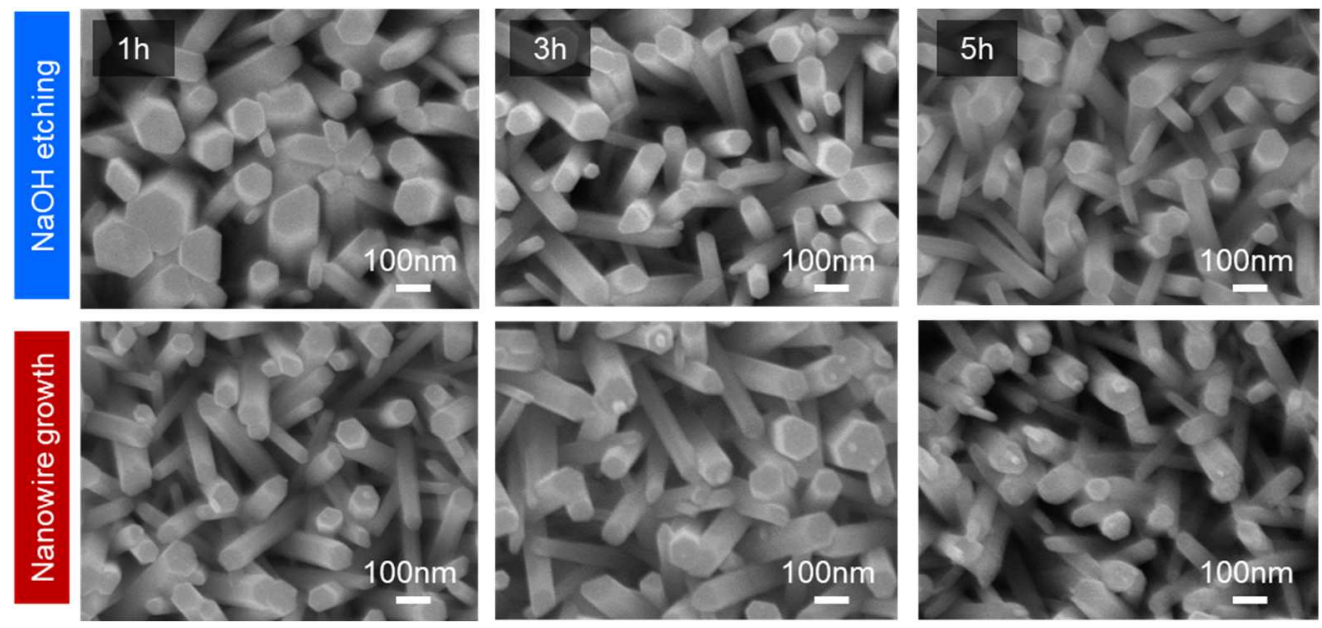

Figure S8. (a) The time-series FESEM images for $\mathrm{NaOH}$-based chemical etching of the $\mathrm{ZnO}$ nanowires (upper) and the results of subsequent nanowire growths (lower). The $\mathrm{pH}$ value of $\mathrm{NaOH}$ solution is controlled to be 11.4 , which is same condition as the ammoniaetching in figure 4 (a) and step 1 in figure 1 (c). In this experiment, the randomly sized $\mathrm{ZnO}$ nanowires with flat-top are first fabricated prior to the chemical etching (Figure S7) and the subsequent nanowire growth is conducted with no $\mathrm{NH}_{3}$ and $C_{Z n} 5 \mathrm{mM}$ for $12 \mathrm{~h}$. Contrary to the ammonia-based etching, the formations of the sharp-tip nanowires and the secondary $\mathrm{ZnO}$ nanowire growths are not observed in case of $\mathrm{NaOH}$-based etching. This indicates that the effect of ammonia-etching observed in figure 4 (a) is due to the ammonia species rather than $\mathrm{pH}$ value. 


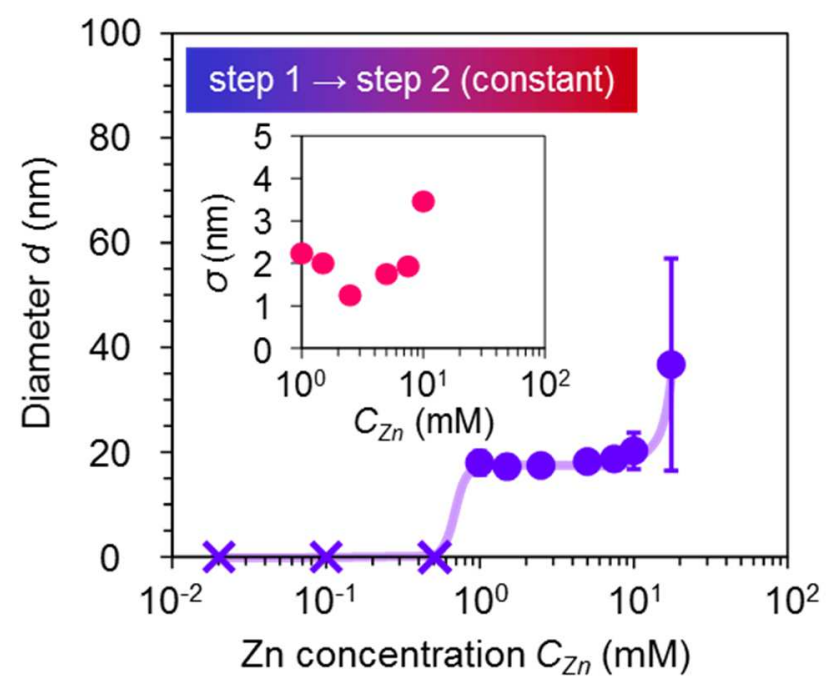

Figure S9. Dependence of the diameter of the two-step grown $\mathrm{ZnO}$ nanowires and the standard deviation (inset) on the $\mathrm{Zn}$ concentration. Only the $\mathrm{Zn}$ concentration in step 1 was varied, and the other conditions were kept constant (with $\mathrm{NH}_{3} 50 \mathrm{mM}$ for step 1, no $\mathrm{NH}_{3}$ and $C_{\mathrm{Zn}}=5 \mathrm{mM}$ for step 2). The growth time for step 1 and step 2 was $3 \mathrm{~h}$ and $12 \mathrm{~h}$, respectively. We found that the diameter and the standard deviation of the two-step grown nanowires were almost unchanged when the $\mathrm{Zn}$ precursor concentration $C_{Z n}$ was varied under the critical concentration for nucleation on $(10 \overline{1} 0)$ plane. This is because the size of (0001) plane is not changed in this condition once the sharp-tip is formed. On the other hand, when increasing $C_{Z n}$ above the critical concentration on (1010) plane, the diameter increases together with the standard deviation value, implying the increase of $(0001)$ plane size. 


\section{Discussion as to the sharp-tip formation by ammonia-etching.}

Considering the difference of the growth conditions between step 1 and step 2, ammonia plausibly affects the sharp-tip formation. Here we consider how ammonia affects the $\mathrm{ZnO}$ formation in the growth process. In our hydrothermal process, the formation of $\mathrm{ZnO}$ can be explained by the following chemical reactions:

$$
\begin{aligned}
& {\left[\mathrm{Zn}\left(\mathrm{NH}_{3}\right)_{n}\right]^{2+}+m \mathrm{OH}^{-} \leftrightarrow \mathrm{Zn}^{2+}+m \mathrm{OH}^{-}+n \mathrm{NH}_{3}} \\
& \leftrightarrow\left[\mathrm{Zn}(\mathrm{OH})_{m}\right]^{2-m}+n \mathrm{NH}_{3} \leftrightarrow \mathrm{ZnO}+\mathrm{H}_{2} \mathrm{O}+(m-2) \mathrm{OH}^{-}+n \mathrm{NH}_{3},
\end{aligned}
$$

where $\mathrm{NH}_{3}$ is supplied through two routes, i.e. the ammonia addition and the decomposition of hexamethylenetetramine (HMTA). In this chemical reaction, the formation and the dissociation of $\mathrm{ZnO}$ simultaneously occur, and the dissociation reaction should be enhanced by adding ammonia, i.e. $\left[\mathrm{Zn}(\mathrm{OH})_{m}\right]^{2-m}$ ions and $\left[\mathrm{Zn}\left(\mathrm{NH}_{3}\right)_{n}\right]^{2+}$ ions increase with increasing $\mathrm{OH}^{-}$ions and $\mathrm{NH}_{3}$ concentrations. In our experiments, the $\mathrm{pH}$ value and the equilibrium concentrations of $\left[\mathrm{Zn}(\mathrm{OH})_{m}\right]^{2-m}$ ions and $\left[\mathrm{Zn}\left(\mathrm{NH}_{3}\right)_{n}\right]^{2+}$ ions become higher when ammonia is added in the growth solution (Figure S6). Since the nanowire top remains flat when the $\mathrm{NaOH}$ solution is employed as an etchant (Figure S8), the sharp-tip formation is caused by the ammonia species and is associated with the formation of $\left[\mathrm{Zn}\left(\mathrm{NH}_{3}\right)_{n}\right]^{2+}$ ions rather than $\left[\mathrm{Zn}(\mathrm{OH})_{m}\right]^{2-m}$ ions. Thus, the sharp-tip are formed by an assist of dissociation reaction in the ammonia added step 1 .

Now we question why the $\{10 \overline{1} 1\}$ planes are preferentially formed during ammonia etching. The energetically unfavourable roughened surface and high Miller index planes immediately disappear at early stage of etching process. In this regard, the lower index 
(0001) and $\{10 \overline{1} 0\}$ planes can survive during the etching. Considering the arrangement of atom in $\mathrm{ZnO}$ crystal, $\{10 \overline{1} 0\}$ planes are composed of successive $\mathrm{Zn}$-aligned and Oaligned polar plane as well as (0001) plane. Since the O-aligned plane is stable and serves as a protective layer for the etchant, the $\{10 \overline{1} 1\}$ planes are able to remain during etching process. On the other hand, the morphology of crystal is configured by the remaining crystal planes and a plane with faster etching rate tends to be exposed when the etching proceeds. Since the ammonia etching is associated with the formation of $\left[\mathrm{Zn}\left(\mathrm{NH}_{3}\right)_{n}\right]^{2+}$ ions, a coordination of $\mathrm{NH}_{3}$ to the $\mathrm{Zn}$ site is of critical importance for the etching. Based on the facts that the $(10 \overline{1} 1)$ plane possesses $\mathrm{Zn}$-aligned polar plane and the number density of in-plane bond for $\mathrm{Zn}$ is less than (0001) plane, the etching rate on (1011) plane must be faster than that of (0001) plane and non-polar (1010) plane. As a consequence, the $\{10 \overline{1} 1\}$ planes remain and the uniformly-shaped tips facetted by the $\{10 \overline{1} 1\}$ planes are deterministically formed during the ammonia etching. 